\title{
Methods of parameterization of amplitudes and extraction of resonances, $D$-decay amplitudes
}

\author{
Robert Kamiński ${ }^{1, *}$ \\ ${ }^{1}$ Institute of Nuclear Physics PAS, Radzikowskiego 152, Kraków
}

\begin{abstract}
Amplitudes used for analyses of two-body interactions very often are not unitary therefore can not guarantee correct results. It is, however, quite easy to construct unitary amplitude or check whether given amplitude fulfills unitarity condition. Only few conditions must be fulfilled to guarantee unitarity. Presently, when in many data analyses very small, overlapping or broad signals are studied, non-unitary effects can significantly influence results and lead to nonphysical interpretation of obtained parameters.
\end{abstract}

\section{Introduction}

Unitarity can be compared to probability that is conserved in nature. Therefore unitarity should play crucial role in analyses of amplitudes in various interactions and decays. Here we concentrate on the simplest case: two-body interactions. The typical easiest way to construct amplitudes containing several resonances is just to add the smallest number of individual amplitudes which sufficiently well describe data. Here we show that amplitudes constructed in such a way may be not unitary and may be not sufficient to describe the data and need additional smooth background. Backgrounds may be interpreted as an effective influence of all omitted singularities of the amplitudes. These singularities usually lie very far from the physical region and are very model dependent, therefore can not be interpreted as real resonances. Resonances which we can find in for example Particle Data Group Tables should be model independent and should play leading role in construction of full amplitudes (phase shifts and inelasticities), both elastic and inelastic. Parameters of resonances (for example mass and width) do, however, depend very much on whether the amplitudes used in analyses were unitary or not.

\section{Unitarity for one resonance amplitude in one channel scattering}

What tell us about unitarity? Similarity between unitarity and probability is difficult to apply directly to amplitude. Square of modulus of amplitude is proportional to cross section for given process and varies with energy. Function which behaves like probability is $S$-matrix (one element in one channel case). Modulus of $S$-matrix is equal to one what strongly constrains analytical structure of unitary amplitude. Let us construct such amplitude for the simplest case - one resonance.

\footnotetext{
*e-mail: robert.kaminski@ifj.edu.pl
} 
Generally $S$-matrix can be expressed as ratio of two Jost functions $S(k)=\frac{D(-k)}{D(k)}=e^{2 i \delta}$. To reproduce resonance in our $S$-matrix let us first assume minimum condition - one zero of the denominator $D(k)$ at $k=k_{j}$. So $D(k)=\left(k-k_{j}\right)$ and $D(-k)=\left(-k-k_{j}\right)$. One can easily check that in such "one pole" case $|S(k)| \neq 1$ therefore our amplitude $A(k)$ related with the $S(k)$-matrix by $A(k)=\frac{S(k)-1}{2 i k}$ is not unitary. Adding second-symmetric pole also on the 2 nd Riemann sheet but at $k=-k_{j}^{*}$ one gets $D(k)=\left(k-k_{j}\right)\left(k+k_{j}^{*}\right)$ and $D(-k)=\left(-k-k_{j}\right)\left(-k+k_{j}^{*}\right)$. One can easily check that now $|S(k)|=1$ and phase shift $\delta=(-\alpha-\beta+\gamma+\omega) / 2$, where $\alpha, \beta, \gamma$ and $\omega$ are phases of the poles $p, p^{\prime}$ and zeroes $z^{\prime}$ and $z$ presented on Fig. (1). Full phase shifts and all components are presented on this figure.

All those phase components are given by $\operatorname{ArcTan}\left(\frac{-I m k_{j}}{k-R e k_{j}}\right)$. It shows that of course only pole $p$ and zero $z^{\prime}$ lying closer to physical region than their mirror pole $p^{\prime}$ and zero $z$ (hereafter "second pole") can produce increase of the phase by $\pi / 2$ what is characteristic for single resonances. Therefore the role of the second pole decreases with the energy. Nowhere is, however, equal to zero. At the threshold, i.e. right in the middle between $p, z^{\prime} p^{\prime}$ and $z$ influence of the all these poles and zeroes on the amplitude is the same.
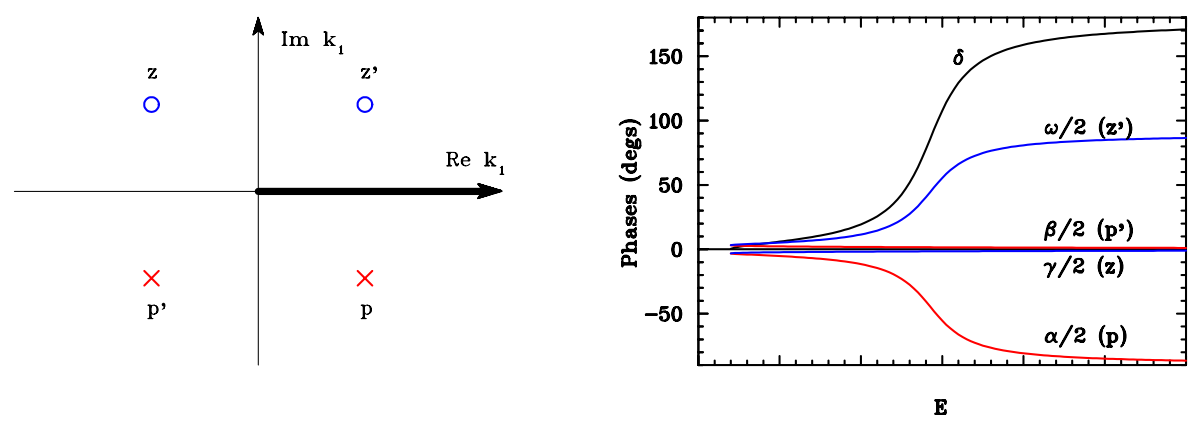

Figure 1. Schematic positions of all necessary poles and zeroes of an unitary amplitude for single resonance in the complex momentum plane (left figure) and phases of all these poles and zeroes compared with their sum $\delta$ - phase shifts of the amplitude (right figure), thick black line denotes physical region.

The most popular amplitude used in for example experimental analyses is Breit-Wigner type amplitude $B W(E)=\frac{\Gamma / 2 k}{M_{B W}-E-i \Gamma / 2}$. Using relation $B W(E)=\frac{S_{B W}(k)-1}{2 i k}$ one can easily calculate $S_{B W}(E)$ and check if this $S$-matrix is unitary as should be. From definition of $B W(E)$ one gets $S_{B W}(E)=\frac{M_{B W}-E+i \Gamma / 2}{M_{B W}-E-i \Gamma / 2}$. One can easily check that $\left|S_{B W}(E)\right|=1$ so single Breit Wigner approximation is unitary. Of course Breit-Wigner is approximation and works well only near the resonance mass, especially for narrow resonances. For example threshold behavior of such amplitude is wrong. In the limit $E \longrightarrow 2 m$ the $S$-matrix $S_{B W}(E) \longrightarrow \frac{2 m-E_{j}^{*}}{2 m-E_{j}}$ (where $\left.E_{j}=M_{B W}-i \Gamma / 2\right)$ and $\delta(E) \longrightarrow \operatorname{ArcTan}\left(\frac{\Gamma / 2}{M_{B W}-2 m}\right) \neq 0$ (also $\sigma(E) \neq 0$ ). In case of unitary $S$-matrix defined by Jost functions with two poles and zeroes presented above, corresponding limits are correct, i.e. $S(k) \longrightarrow \frac{-k_{j} * k_{j}}{-k_{j} * k_{j}}$ so $\delta(k) \longrightarrow 0$ and $\sigma(k) \longrightarrow 0$.

\section{Unitarity for amplitude with more resonance and more channels}

For amplitudes with more resonances very popular is isobar model with sum of amplitudes describing, for example, single resonances. For two amplitudes, their sum $T_{1,2}=T_{1}+T_{2}=$ 
$\frac{S_{1}-1}{2 i k}+\frac{S_{2}-1}{2 i k}$ what corresponds to $S_{1}+S_{2}=e^{2 i \delta_{1}}+e^{2 i \delta_{2}}$. Of course $\left|S_{1}+S_{2}\right| \neq 1$ what means that isobar model violates unitarity. In case of analyses using $S$-matrix instead of amplitudes one can create product $S_{1} S_{2}$ which fulfills unitarity (for more resonances described by $S_{i}$ the method is the same). Another popular way of parameterization of amplitudes is to use $K$-matrix defined by $S=(1+i K) /(1-i K)$. Sum of two $K$-matrices does not violate unitarity.

In case of more that one channel situation becomes more complicated. Because of simple ambiguity $k_{2}= \pm \sqrt{k_{1}^{2}+m_{1}^{2}-m_{2}^{2}}$ every new channel doubles number of Riemann sheets. So in case of $n$ channels one has $2^{n}$ Riemann sheets and $2^{n-1}$ poles lying on various Riemann sheets and coming from one single pole appearing in one of the channels in fully decoupled case. All these poles are shifted more or less (it depends on strength of coupling between channels) with respect to position of this original pole.

Figure (2) presents Riemann sheets for two channels and schematic positions of poles and zeroes corresponding to one resonance. Names of Riemann sheets are given by signs of imaginary parts of momenta in all channels. For example in two channel case mark $(-,+)$ means that $\operatorname{Im}\left(k_{1}\right)<0$ and $\operatorname{Im}\left(k_{2}\right)>0$

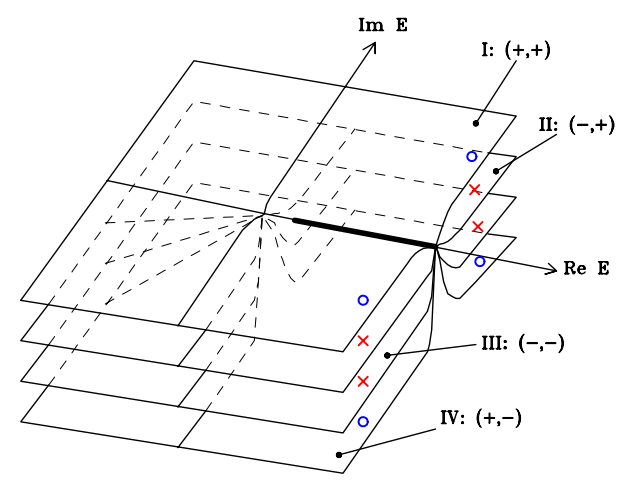

Figure 2. Riemann sheets for two channels with poles (crosses) and zeroes (circles) for one resonance

Table 1 presents an example of positions of the $S$-matrix poles for three resonances found in two channel analysis of scalar-isoscalar $\pi \pi$ interactions below $2 \mathrm{GeV}$ (analysis similar to that in [1]). Underlined are poles which play leading role in the full amplitude. They were found checking distances of the poles from the physical region in complex conformal variable $z$ defined by $z=\frac{k_{1}+k_{2}}{\sqrt{m_{K}^{2}-m_{\pi}^{2}}}$. The results of such analysis have been confirmed by analysis of phases and squared modules of amplitudes (proportional to cross section) corresponding to each pole. Figures (3) and (4) present phases and squared modules of amplitudes for each pole separately and for pairs of poles related with given resonance. It is seen that in both cases (especially for $f_{0}(980)$ ) one pole is dominant and the second one plays minor role.

In case of analysis of more than 2 channels one can not define and use similar conformal variable and the simplest and effective method is just analysis of influence of all found poles on the phase shifts and inelasticities as was shown on Figs (3) and (4). Another method relays on presentation of positions of all poles in 3 dimensional combinations of real and/or 
Table 1. Positions of two channel $S$-matrix poles found in analysis of scalar-isoscalalr $\pi \pi$ interactions below $2 \mathrm{GeV}$

\begin{tabular}{|c|c|c|c|}
\hline Pole & $R e E_{\text {pole }} \mathrm{MeV}$ & $I_{m} E_{\text {pole }} \mathrm{MeV}$ & R. sheet \\
\hline 1 & 639.6 & -323.9 & $(-,-): I I I$ \\
$\frac{1}{2}$ & $\frac{511.4}{982.0}$ & $\frac{-230.6}{-36.9}$ & $\frac{(-,+): I I}{(-,+): I I}$ \\
$\frac{2}{2}$ & $\frac{-8.4}{432.4}$ & $\frac{-79.3}{(-,-): I I I}$ & $\frac{(-,-): I I I}{(-,+): I I}$ \\
$\frac{3}{3}$, & $\frac{1431.7}{1394.9}$ & -120.6 & \\
\hline
\end{tabular}
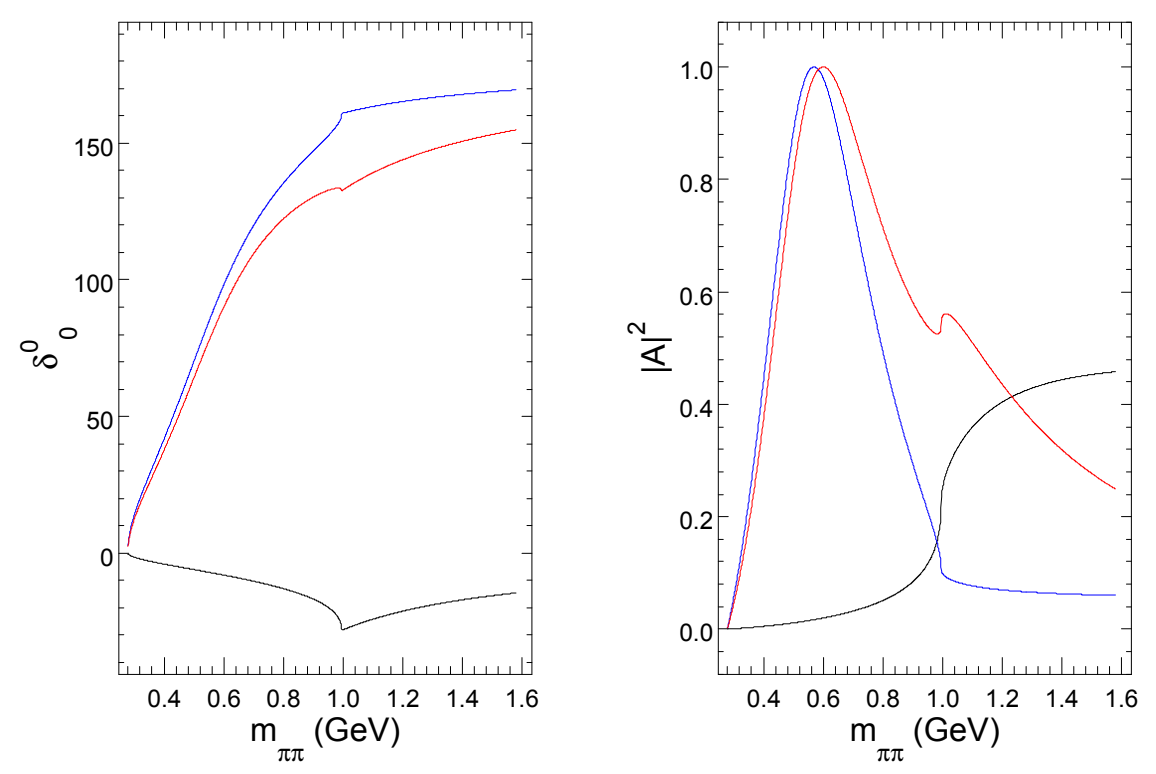

Figure 3. Phase shifts and squared modules of amplitudes produces by single poles from Table 1 and their pairs, black lines are for pole 1, blue for 1' and red for both poles together.

imaginary parts of complex momenta in all channels. Reasonable choice of axis and careful analysis of distances of these poles from physical region enables to identify the most prominent poles. Example of results of such 3-channel analysis one can find in [2] (Tables 3-7).

Independently on analyzed number of channels and number of found resonances crucial is the use of correct i.e unitary amplitude. In recent analysis of pion electromagnetic form factor [3] authors present various ways of parameterization of $e^{+} e^{-} \rightarrow \pi^{+} \pi^{-}$cross section and of vector-isoscalar $\pi \pi$ elastic amplitude. In Table 2 compared are parameters of $\rho$ states obtained using Gounaris-Sakurai model and unitary and analytic approach. The latter one gives significantly different results than those from PDG Tables and than obtained using GounarisSakurai model. Mass difference for $\rho(770)$ is about $9 \mathrm{MeV}$ and for $\rho^{\prime}$ and $\rho^{\prime \prime}$ about $170 \mathrm{MeV}$ and $78 \mathrm{MeV}$ respectively. Sign of these differences agrees with what was presented in Section 2 (mass from unitary amplitude is smaller). Small phase produced by second pole (denoted in Section 2 by $p^{\prime}$ ) leads to shift of the main pole $p$ (i.e. shift of the mass) to lower energies 

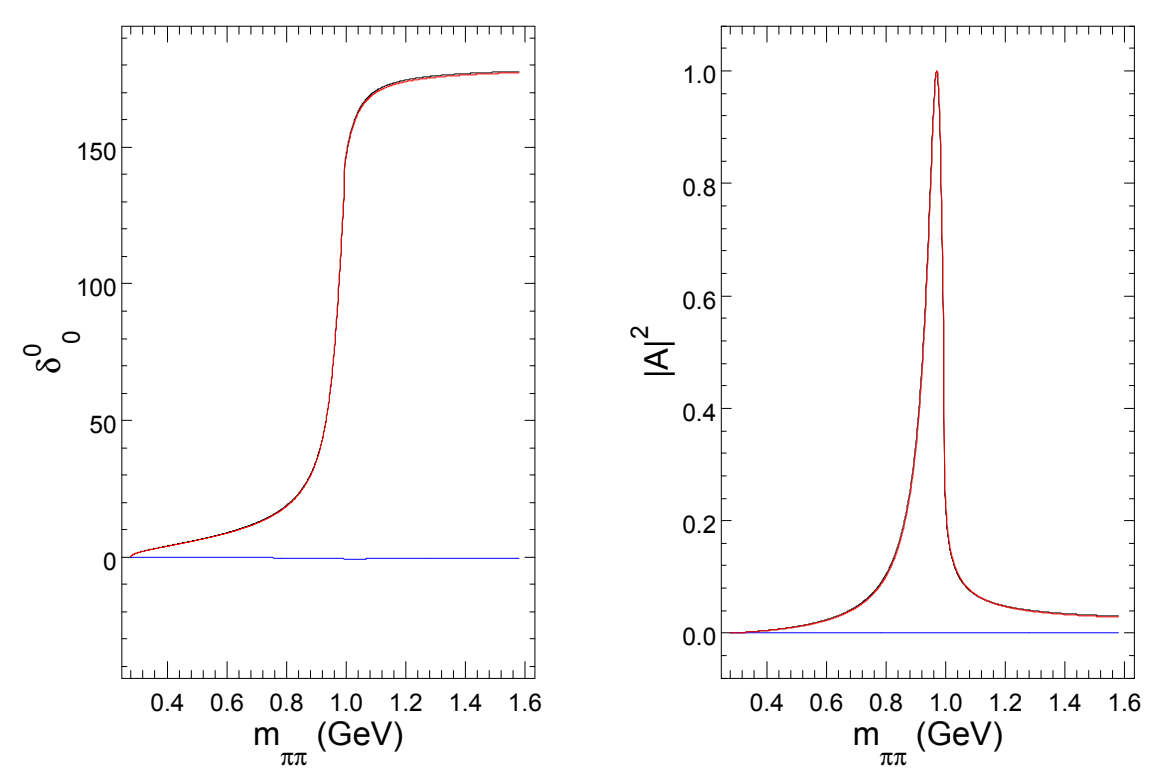

Figure 4. Phase shifts and squared modules of amplitudes produces by single poles from Table 1 and their pairs, black lines are for pole 2, blue for 2' and red for both poles together.

Table 2. Parameters of $\rho$ states obtained using Gounaris-Sakurai model and unitary and analytic approach are compared with values from Particle Data Tables [4].

\begin{tabular}{|c|c|c|c|}
\hline Parameter & PDG MeV & G.S. MeV & U\&A MeV \\
\hline$m_{\rho}$ & $775.26 \pm 0.25$ & $774.81 \pm 0.01$ & $763.88 \pm 0.04$ \\
$m_{\rho^{\prime}}$ & $1465.00 \pm 25.00$ & $1497.70 \pm 1.07$ & $1326.35 \pm 3.46$ \\
$m_{\rho^{\prime}}$ & $1720.00 \pm 20.00$ & $1848.40 \pm 0.09$ & $1770.54 \pm 5.49$ \\
$\Gamma_{\rho}$ & $149.10 \pm 0.80$ & $149.22 \pm 0.01$ & $144.28 \pm 0.01$ \\
$\Gamma_{\rho^{\prime}}$ & $400.00 \pm 60.00$ & $442.15 \pm 0.54$ & $324.13 \pm 12.01$ \\
$\Gamma_{\rho^{\prime \prime}}$ & $250.00 \pm 100.00$ & $322.48 \pm 0.69$ & $268.98 \pm 11.40$ \\
\hline
\end{tabular}

in comparison with mass determined by value of phase shift equal to $\pi / 2$. In case of $\rho(770)$ this shift should be few $\mathrm{MeV}$ and for wider states should be bigger what agrees with numbers in Table 2.

\subsection{Crossing symmetry as additional constraint}

It is very advisable to introduce the crossing symmetry requirement to the amplitudes. For identical particles like $\pi \pi$ it quite easy and was proposed by Roy few decades ago [5] and later was developed and applied in number of works in the early 2000s e.g. [6] and [7]. For non-identical mesons like $\pi$ and $K$ similar analysis was performed recently [8].

Introduction of the crossing symmetry requirement to amplitudes describing scalarisoscalar $\pi \pi$ interactions has led to spectacular successes. One of them was to eliminate the long standing up-down ambiguity in these amplitudes (in favor of the down solution) 
[9] and the second was to reduce by factor about 6 the uncertainty caused by the significant dispersion of experimental data. This reduction was possible thanks to the application of a newly derived set of Roy-type dispersion relations GKPY with one subtraction [7].

\subsection{Example of unitary amplitudes in heavy meson decays}

Heavy meson decays are very often described using popular isobar model. For example in one of the BaBar analysis of the $D^{0} \rightarrow K_{S}^{0} K^{+} K^{-}$decays [10] isobar model with $A_{\text {tot }}(\mathbf{m})=$ $\sum_{r} a_{r} e^{i \Phi_{r}} A_{r}(\mathbf{m})+a_{N R} e^{i \Phi_{N R}}$ was used for 5 resonances: $a_{0}(980), a_{0}(1450), f_{0}(1370), \phi(1020)$ and $f_{2}(1270)+$ their charge "twins". In results sum of the fraction was $152.2 \%$ and $\chi^{2}=$ 1.1 pdf for 14 free parameters.

Using a different method to describe these decays (based on quasi-two-body factorization) the total amplitude may be expressed by $A_{t o t}(m)=S_{0}(m)+S_{+}(m)+S_{-}(m)+P_{0}(m)+D_{0}(m)$ with unitary $S_{0}(m), S_{+}(m), S_{-}(m), P_{0}(m)$ and $D_{0}(m)$ components. In results sum of the fraction is $\approx 130 \%$ and $\chi^{2} \approx 1.2$ pdf for 16 free parameters [11]. The same method with also unitary amplitudes in single channels has already been successfully used to describe the decays of other heavy mesons (see for example [12]).

\section{Conclusions}

The condition of unitarity is very important and quite easy to introduce when building amplitudes. They must meet several conditions, such as for example the presence of two symmetrical poles for each resonance. When drawing conclusions from the analysis of multi-channel amplitudes fitted to the experimental data, it is extremely important to correctly identify the leading poles and link them to existing (or new) resonances. To avoid ambiguity in determining the parameters of these resonances, it should always be clearly explained how they were identified and what amplitudes were used for this.

\section{Acknowledgments}

The work was created as a result of the implementation of the Polish research project No. 2018/29/B/ST2/02576 financed from the funds of the National Science Center

\section{References}

[1] R. Kamiński, L. Leśniak and J.-P. Maillet, Phys. ReV. D50, 3145 (1994).

[2] R. Kamiński, L. Leśniak and B. Loiseau, Eur. Phys. J. C9, 141 (1999).

[3] Erik Bartoš et al., Phys. Rev. D96, 113004 (2017).

[4] C. Patrignani et al. (Particle Data Group Collaboration), Chin. Phys. C 40, 100001 (2016).

[5] S. M. Roy, Phys. Lett. 36B, 353 (1971).

[6] G. Colangelo, J. Gasser and H. Leutwyler, Nucl. Phys. B603, 125 (2001); B. Ananthanarayan, G. Colangelo, J. Gasser and H. Leutwyler, Phys. Rep. 353, 207 (2001).

[7] R. García-Martín et al., Phys. ReV. D83, 074004 (2011).

[8] J. R. Pelaez, A. Rodas and J. Ruiz de Elvira, Eur. Phys. J. C77, 91 (2017); J. R. Pelaez and A. Rodas, Phys. reV. D93, 074025 (2016); J. R. Pelaez and A. Rodas, Eur. Phys. J. C78, 897 (2018).

[9] R. Kamiński, L. Leśniak and B. Loiseau, Phys. Lett. B551, 241 (2003).

[10] B. Aubert et al. (BABAR Collaboration), Phys. Rev. D 78, 034023 (2008).

[11] Temporary results, works in progress.

[12] D. Boito et al., Phys. Rev. D96, 113003 (2017). 